\title{
APPROACHES FOR IMPROVED DOPPLER ESTIMATION IN LIDAR REMOTE SENSING OF ATMOSPHERIC DYNAMICS
}

\author{
Sreevatsan Bhaskaran and Ronald Calhoun \\ Environmental Remote Sensing Group, Arizona State University, AZ 85281, USA, *Email: \\ sbhaska4@asu.edu
}

\begin{abstract}
Laser radar (Lidar) has been used extensively for remote sensing of wind patterns, turbulence in the atmospheric boundary layer and other important atmospheric transport phenomenon. As in most narrowband radar application, radial velocity of remote objects is encoded in the Doppler shift of the backscattered signal relative to the transmitted signal. In contrast to many applications, however, the backscattered signal in atmospheric Lidar sensing arises from a multitude of moving particles in a spatial cell under examination rather than from a few prominent "target" scattering features. This complicates the process of extracting a single Doppler value and corresponding radial velocity figure to associate with the cell. This paper summarizes the prevalent methods for Doppler estimation in atmospheric Lidar applications and proposes a computationally efficient scheme for improving Doppler estimation by exploiting the local structure of spectral density estimates near spectral peaks.
\end{abstract}

\section{INTRODUCTION}

Estimation of range rate by measuring Doppler shift of scattered signals is a foundational capability in radar remote sensing of dynamic scenes. In the context of atmospheric sensing with laser radar, the backscattered signal from a single range cell results from interaction of the transmitted light with a large number of particles in the cell. Each of these will have its own velocity, and a typical objective is to deduce a mean range rate from the superposition of these backscattered signals to describe the aggregate atmospheric motion within the cell.
Most current-generation commercial lidars estimate Doppler using straightforward digital signal processing methods such as the Periodogram Discrete spectral Peak Estimator [1] or the Maximum Likelihood Estimator described in [2]. Both of these methods begin by forming a discrete spectral density estimate from digitally sampled temporal data representing the range cell of interest and then identifying a single dominant peak in this spectrum estimate. The (discrete) frequency of this peak is accepted as the Doppler frequency from which a singly radial velocity representing the entire cell is calculated.

In view of the high data flow rate in Lidar systems, it is important that the signal processing algorithms be computationally efficient as well as accurate. For Doppler frequency estimation, accuracy of an unbiased estimator may be characterized by its variance, which is desirable to bring as close as possible to the Cramér-Rao lower bound [2].

In this paper we examine methods for Doppler estimation, inspired by [4], that exploit a small neighborhood of values around the maximal peak in the spectral density estimate to improve estimation accuracy relative to accepting the frequency of the single peak as the estimate. Tradeoffs between additional computational complexity entailed in these methods and the improvement in estimation accuracy they provide are quantified, and their performance is compared to performance descriptors for other algorithms that appear in [2].

\section{METHODOLOGY}

We use a standard periodogram spectrum estimator based on the Discrete Fourier Transform (DFT) to estimate the spectrum from multiple blocks of time series data corresponding to a 
given range cell. This approach intrinsically produces spectral density estimates at a discrete set of frequencies ("bins"), the spacing of which (in Hertz) is determined by the duration (in seconds) of the time series segments of which DFTs are taken. Choosing a single peak from this discrete spectrum estimate to represent the Doppler frequency inherently limits the frequency resolution to be no finer than the bin spacing of the DFT. The method presented here affords the possibility of a Doppler estimate that falls between two DFT bins by performing an interpolation that uses a small number of periodogram values on each side of the peak and considers the spectral shape of the DFT bins in a mathematically appropriate way. The computational complexity of this approach is seen to exceed the single-peak method by an amount that increases with the number of neighboring values used in the interpolation; it is small when small local neighborhoods of the peak are used.

It is noteworthy that the Doppler estimation problem in Lidar sensing of atmospheric dynamics differs significantly from the classical problem of estimating the frequency of a tone in noise. This is observed in actual Lidar data and also in data simulated from standard models $[5,6]$, such as shown in Figure 1. The Doppler signal is not tonal, but has numerous frequency components arising from the diversity of scatterers within the range bin. When the aggregate velocity of these particles is well defined, the spectral energy is correspondingly concentrated, but rarely within a single bin of the periodogram. There is also uncertainty associated with the time-frequency bandwidth limitation, which adds to the spread of the peak power over multiple spectral bins.

This characteristic of the data provides further rationale for considering more than a single peak in arriving at a figure to represent the aggregate radial velocity of the flow in a range cell. Our approach builds on the method for estimating the frequency of a tone in noise given in [4] and shown to yield estimation error variance on the same order as the Cramér-Rao bound. We adapt from the tonal setting to accommodate the frequency spread observed in lidar Doppler estimation and we describe the performance in terms of Cramer-Rao efficiency assuming a mathematical model of the frequency spread.

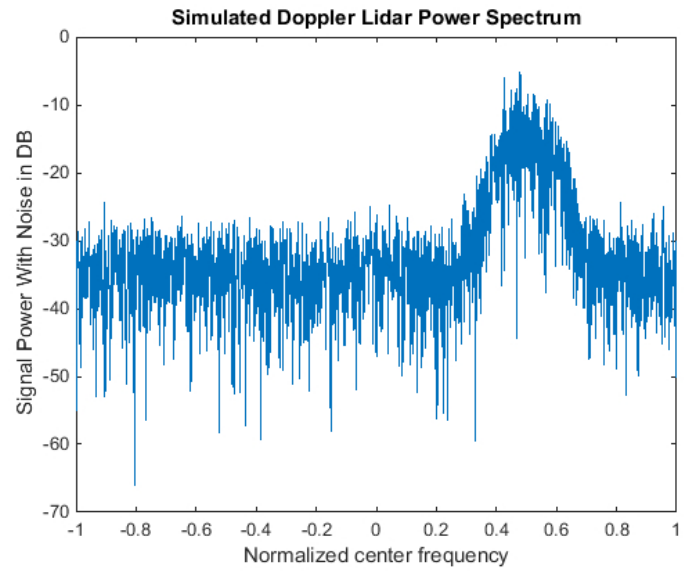

Fig.1 Simulated ${ }^{5,6}$ Doppler Lidar Spectrum, Sampling time $\mathrm{T}=0.5 \mathrm{sec}, \mathrm{SNR}=10 \mathrm{~dB}$, Spectral Width of signal $\sigma=0.625$. Demonstrates the smeared peak frequency observed on the frequency spectrum, over multiple channels.

\section{RESULTS}

The paper aims at presenting an exploratory technique, tested on Lidar returns. The localized point complex interpolation technique, is analyzed both in terms of its estimation fidelity and computational complexity. A detailed feasibility study of this technique, and comparison with the two most prominent estimator algorithms specified in the introduction, is required to completely justify the use of the new estimator algorithm for Lidar returns.

\section{CONCLUSION}

The technique suggested here is a promising motivation to improve the uncertainty associated with the peak estimation problem in a Lidar. Simultaneously, we would also need to address the necessity to process the huge data flow rate in a Lidar. A possible contender in this area is using neural network approaches. This would be an exploratory future direction, to address the huge data flow rate problem and to optimize the speed of the signal processing algorithms used in a Lidar system 


\section{REFERENCES}

[1] B. J. Rye and R. M. Hardesty, "Discrete spectral peak estimation in incoherent heterodyne backscatter Lidar I - Spectral estimation and Cramér-Rao lower bound," IEEE Trans. Geosci. Remote Sens., 31, no. 1, January 1993.

[2] R. G. Frehlich and M. J. Yadlowsky, "Performance of mean frequency estimators for Doppler radar and Lidar," J. Atmos. Ocean Tech., Vol. 11.

[3] D. S. Zrinc, "Simulation of weather like Doppler spectra:," Journal of Applied Meteorology, 14, June 1975.

[4] B. G. Quinn, "Estimating frequency by interpolation using Fourier coefficients," IEEE Trans. Signal Processing, 42, no. 5, May 1994. 